\title{
Pneumocephalus Secondary to Pneumomedias Tinum, Pneumohemothorax and Subcut Aneous Emphysema
}

\author{
${ }^{1}$ A.A. Akbari Kamrani, ${ }^{2} \mathrm{~B}$. Khorasany, ${ }^{3} \mathrm{M}$. Nabiyouni, ${ }^{4} \mathrm{M}$. Khorasani and ${ }^{5} \mathrm{M}$. Ghafoori \\ ${ }^{1}$ Iranian Research Center on Aging, \\ University of Social Welfare and Rehabilitation Sciences, Tehran, Iran \\ ${ }^{2}$ University of Social Welfare and Rehabilitation Sciences, Tehran, Iran \\ ${ }^{3}$ Payambaran Hospital, Tehran, Iran \\ ${ }^{4}$ Qazvin University of Medical Sciences, Qazvin, Iran \\ ${ }^{5}$ University of Medical Sciences, Tehran, Iran
}

\begin{abstract}
Researchers report a case of pneumocephalus secondary to pneumomediastinum and pneumothorax and subcutaneous emphysema as a result of car accident. About 55 years old man with a head and neck trauma and right upper limb paralysis shown to the emergency department without any evidence of skull fracture. About 8 days later he developed symptoms of neurologic deterioration. Multislice CT scanning of the head showed extensive pneumocephalus adjacent to right. Frontal lob with pressure effect on underlying brain parenchyma and shift of brain, ventricles and midline structures toward left side spiral lung CT scan showed penumomedistinum, extensive subcutaneous emphysema in the Right hemi thorax, mild pneumothorax and irregularity in the first rib in right side. After 2 weeks of follow-up, with conservative treatment, the intracranial air resolved spontaneously and the patient completely recovered.
\end{abstract}

Key words: Pneumocephalus, pneumomediastinum, pneumohemothorax, subcutaneous emphysema, Iran

\section{INTRODUCTION}

Case report: A 55 years old man that who had a car accident with acute head and neck trauma shown to the emergency department. He shows a few minutes of the unconsciousness immediately after the car accident and complained of pain and tenderness of the first rib in the right side and moving disability of the right upper limb.

Physical examination revealed a laceration and a wound on the left side of the frontal skin as long as $6 \mathrm{~cm}$. He was alert and fully oriented and he had Glasgow Coma Scale score of 15 and there were no physical signs of raised intracranial pressure and there were not any evidence of skull fracture. The patient had paralysis of right upper limb that it seemed due to root avulsion. The patient's past medical history was remarkable for diabetes. Chest X-ray showed elevated diaphragm in right hemithorax. Facial radiographics showed mandiular and zygomatic fractures that treated by maxillofacial surgeon.

\section{MATERIALS AND METHODS}

About 3 days later, a chest tube was placed for drainage of hemothorax in right hemithorax and 4 days later, it removed. The next day of chest tube removal, he developed the symptoms of cerebral compression with unconsciousness and incontinence.

Multislice computed tomography scanning of the head showed extensive pneumocephaus more prominent adjacent to right frontal lob with pressure effect on underlying brain parenchyma and shift of brain, ventricles and midline structures toward left side.

Minimal air was seen in the frontal horn of right lateral ventricle, subcutaneous emphysema was seen in right occipital region. Bony structures and visible vessels were intact and all ventricles showed normal size (Fig. 1a and b). Spiral multislice lung CT scan without contrast showed: pneumomediastinum with no evidence of midline shift, extensive subcutaneous emphysema in the soft tissues of right hemi thorax, irregularity in the first rib in right side mild pneumothorax and pleural effusion in right hemi thorax and athelectasis in right lower lob (Fig. 2a, b and 3a, b).

Routine laboratory test finding were normal. After 2 weeks of follow-up with conservative treatment, the intracranial air, resolved spontaneously and the patient completely recovered and referred to neurosurgeon for repairing of root avulsion (Fig. 3a and b).

Corresponding Author: B. Khorasany, University of Social Welfare and Rehabilitation Sciences, Tehran, Iran 

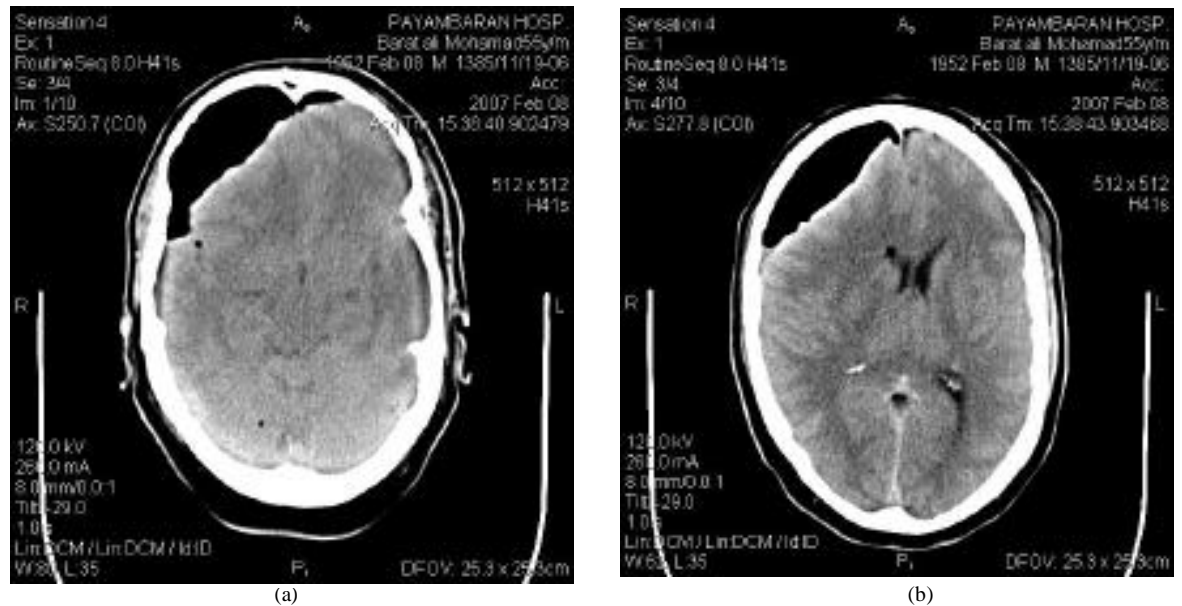

Fig. 1: Bony structure and visible vessels (a) intact and (b) venrticles

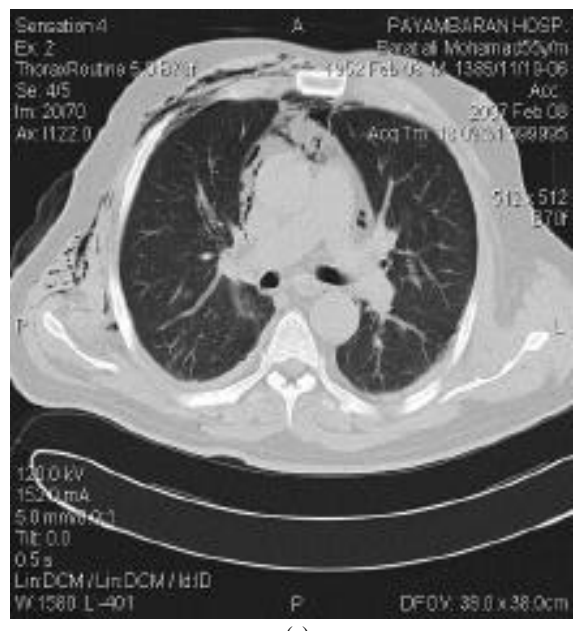

(a)

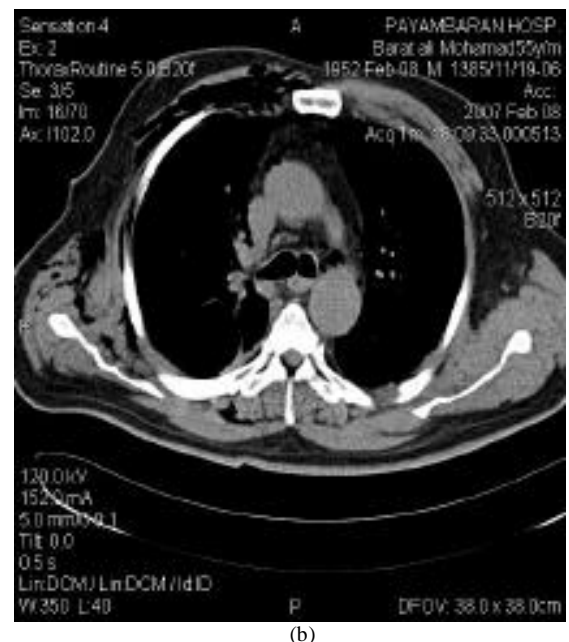

Fig. 2: Mild pneumothorax and pleural effusion in (a) right hemi thorax and (b) althelectasis in right lower lobe
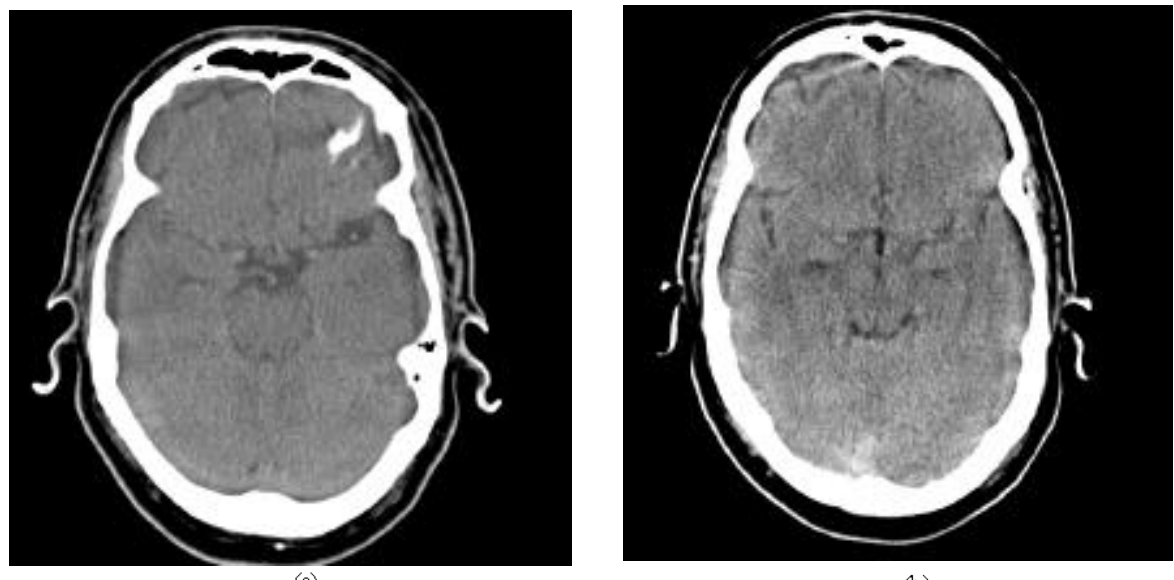

(b)

Fig. 3: Intracranial air resolved and patient reffered to neurosurgeon for $(a, b)$ repairing of root avulsion 


\section{RESULTS AND DISCUSSION}

The incidence of pneumocephalus is shown to be between 0.5 and $1 \%$ of patients with a traumatic brain injury (Orebaugh and Margolis, 1990) and increase to 10 fold in patients with basilar skull fracture (Lewin, 1954). Acute traumatic pneumocephalus is diagnostic of a basilar skull fracture whereas late pneumocephalus which appears a few days post injury is suggestive of a cerebrospinal fluid fistula (Gautschi and Zellweger, 2006).

A pneumocephalus typically causes a sudden head ache (Ash et al., 1991) and it may manifest symptoms and signs ranging from a persistent headache, lethargy, confusion and hemiplegia (Katz et al., 1990). Pneumocephalus may produce mass effect and typical symptoms include: increased intracranial pressure (e.g., vomiting, nausea, headache) and impaired consciousness. Other neurological signs including: seizures, visual field defect and behavioral changes (North, 1971).

The main constituent of the intracranial air collection is nitrogen. Increasing the inspired oxygen concentration reduced the partial pressure of nitrogen in the blood creating a greater concentration gradient for nitrogen to diffuse into the blood stream (Dexter and Reasoner, 1996). Therefore, use of a high inspired air/oxygen concentration can hasten absorption of intracranial air collection (Becker, 2002).

Long-term sequel from pneumocephaus has not been shown. But rare, in tension pneumocephalus, the volume of intracranial air places pressure on the brain and cause herniation and ultimately death if not recognized and treated with surgical evacuation of the trapped air pocket (Guarino and Wright, 2005). Although, neurological deterioration has been associated with pneumocephalus of volumes as low as $25 \mathrm{~mL}$ of air under tension. Oliver P. Gautschi has shown a young patient would remain asymptomatic with an extent of pneumocephalus.

In this asymptomatic case, the patient was managed conservative with bed rest and $100 \%$ oxygen therapy to wash out nitrogen and decrease intracranial gas (Sherman and Bokhari, 2003).

\section{CONCLUSION}

The case report conclude a late pneumocephalus without any skull fracture, 8 days after a car accident and one day after removing the chest tube. It seems after removal the chest tube; pneumothorax, pneumomediastinum and subcutaneous emphysema increased and arrived into the intracranial space from a nerve root for a men and resulted in pneumocephalus. Although, this pneumocephalus produced mass effect and deterioration in neurological functions but resolved spontaneously with conservative treatments.

\section{REFERENCES}

Ash, K.M., J.E. Cannon and D.R. Biehl, 1991. Pneumocephalus following attempted epidural anesthesia. Can. J. Anesthesia, 38: 772-774.

Becker, W.J., 2002. Pneumocephalus as a cause for head ache. Can. J. Neurol. Sci., 29: 278-281.

Dexter, F. and D.K. Reasoner, 1996. Theoretical assessment of normobaric oxygen therapy to treat pneumocephalus. Anesthesiology, 84: 442-447.

Gautschi, O.P. and R. Zellweger, 2006. Asymptomatic posttraumatic pneumocephalus. Am. J. Emergency Med., 24: 628-629.

Guarino, A.H. and N.M. Wright, 2005. Pneumocephalus after a lumbar epidural steroid injection. Pain Phys., 8: 239-241.

Katz, Y., R. Markovits and B. Rosenberg, 1990. Pneumoencephalus after inadvertent intrathecal injection during epidural block. Anesthesiology, 73: $1277-1279$.

Lewin, W., 1954. Cerebrospinal fluid rhinorrhea in closed head injuries. Br. J. Surgery, 42: 1-18.

North, J.B., 1971. On the importance of intracranial air. Br. J. Surgery, 58: 826-829.

Orebaugh, S.L. and J.H. Margolis, 1990. Post-traumatic intracerebral pneumatocele: A case report. J. Trauma, 30: $1577-1580$.

Sherman, C.S. and F. Bokhari, 2003. Massive pneumocephalus after minimal head trauma. J. Emergency Med., 25: 319-320. 